\title{
Oropharyngeal Oxygen Concentration Is Dependent on the Oxygen Mask System and Sampling Location
}

\author{
N David Yanez, Alexander Y Fu, Miriam M Treggiari, and Jeffrey R Kirsch
}

\begin{abstract}
BACKGROUND: Numerous oxygen delivery systems are used to treat hypoxemia. It is unknown if $\mathrm{F}_{\mathrm{IO}_{2}}$ at the lips predicts oropharyngeal $\mathrm{F}_{\mathrm{IO}_{2}}$ for various oxygen mask systems. We tested whether $\mathrm{F}_{\mathrm{IO}_{2}}$ measurements differed between the lips and oropharynx, and whether this difference depends on the mask system. METHODS: Ten healthy volunteers had one sampling catheter positioned at the lips and another catheter in the oropharynx. $\mathrm{F}_{\mathrm{IO}_{2}}$ was sampled at each location while the subjects breathed normal tidal volumes with oxygen at $15 \mathrm{~L} / \mathrm{min}$ via 4 delivery devices: a simple mask, a non-rebreather mask, a face mask with a diffuser that concentrates and directs $\mathrm{O}_{2}$ toward the mouth and nose (mask with diffuser), and a closed mask with a Jackson-Rees circuit. Data were analyzed by using a linear mixed model to account for subject crossover in the repeated measures design. RESULTS: $\mathrm{F}_{\mathrm{IO}_{2}}$ levels differed significantly for the 4 delivery mask systems $(P<.001)$ and by sampling catheter location $(P<.001)$. Differences in mean $\mathrm{F}_{\mathrm{IO}_{2}}$ between the lips and the oropharynx were observed for the mask with diffuser (difference $0.30,95 \% \mathrm{CI} 0.25-0.36 ; P<.001$ ), and non-rebreather mask (difference $0.09,95 \%$ CI $0.04-0.15 ; P=.001$ ). The mean $\mathrm{F}_{\mathrm{IO}_{2}}$ at the oropharynx was highest for the closed mask $(0.97,95 \%$ CI $0.92-1.00)$, followed by the non-rebreather mask (0.76, 95\% CI 0.72-0.81), simple mask (0.62, 95\% CI 0.58-0.67), and the mask with diffuser $\left(0.51,95 \%\right.$ CI 0.46-0.56). At the lips, the mean $\mathrm{F}_{\mathrm{IO}}$, was highest for the closed mask $(0.97$, 95\% CI 0.92-1.00), followed by the non-rebreather mask (0.86, 95\% CI 0.81- 0.90), OxyMask (0.81, 95\% CI 0.76-0.86), and simple mask (0.67, 95\% CI 0.62-0.71). CONCLUSIONS: With high oxygen flows and normal tidal volume breathing, $\mathrm{F}_{\mathrm{IO}_{2}}$ measurements obtained at the oropharynx or at the lips depended on the device used, with the mask with diffuser showing the most significant discrepancies. $\mathrm{F}_{\mathrm{IO}_{2}}$ measures at the oropharynx and the lips were only consistent for the closed mask system. (ClinicalTrials.gov registration NCT02523586.) Key words: hypoxemia prevention and control; oxygen inhalation; therapy; respiratory therapy; perioperative; critical care; respiratory insufficiency; equipment design. [Respir Care 2020;65(1):29-35. (C) 2020 Daedalus Enterprises]
\end{abstract}

\section{Introduction}

In today's perioperative and critical care setting, various oxygen delivery devices are used to treat or prevent hy-

Dr Yanez is affiliated with the Division of Biostatistics, School of Public Health, Oregon Health and Science University, Portland, Oregon. Dr Fu is affiliated with Anesthesiology, El Camino Hospital, Mountain View, California. Drs Treggiari and Kirsch are affiliated with the Department of Anesthesiology and Perioperative Medicine, School of Medicine, Oregon Health and Science University, Portland Oregon.

The authors have disclosed no conflicts of interest.

Correspondence: N David Yanez PhD, Oregon Health and Science University, Oregon Health and Science University/PSU School of Public poxemia according to their reported ranges of $\mathrm{F}_{\mathrm{IO}_{2}}$ (Table 1). ${ }^{1,2}$ Certain clinical scenarios, such as impending respiratory failure and preoxygenation before intubation, require delivery of high $\mathrm{F}_{\mathrm{IO}_{2}}$. Especially in these situations, it is critical that the reported $\mathrm{F}_{\mathrm{IO}_{2}}$ range delivered by the chosen oxygen delivery device is accurate. Studies described gas sampling at the oropharynx, nasopharynx, hypopharynx, and trachea to determine $\mathrm{F}_{\mathrm{IO}_{2}}$ for oxygen delivery devices. ${ }^{2-6}$ The second-generation OxyMask

Health, Mailcode GH-230, 3181 SW Sam Jackson Park Rd, Portland, OR 97239. E-mail: yanezn@ohsu.edu.

DOI: $10.4187 /$ respcare. 07027 
(SouthMedic, Barrie, Ontario, Canada) is an open-faced mask with an oxygen diffuser directed at the nose and mouth, reported to deliver $\mathrm{F}_{\mathrm{IO}_{2}}$ of up to 0.90 (mean 0.80) at $15 \mathrm{~L} / \mathrm{min}$ based on gas sampled at the lips. ${ }^{2,7}$ However, there is no evidence that $\mathrm{F}_{\mathrm{IO}_{2}}$ measured at the lips is accurate compared with $\mathrm{F}_{\mathrm{IO}_{2}}$ deeper in the airway. Because sampling at the lips does not account for nasal breathing of a different oxygen concentration or gas mixing inside the mouth and pharynx due to entrained air, it is unclear if $\mathrm{F}_{\mathrm{IO}_{2}}$ at the lips accurately reflects $\mathrm{F}_{\mathrm{IO}_{2}}$ that reaches the lungs. If nasal breathing or gas mixing indeed results in a difference between measurements at the lips and measurements at locations deeper in the airway, one would expect this difference to be more pronounced for oxygen masks with more-open designs, for example, the OxyMask, because of more potential for gas mixing.

Theoretically, tracheal sampling would provide the most accurate representation of the gas mixture participating in gas exchange in the lungs. Although the use of a tracheal catheter for measuring $\mathrm{F}_{\mathrm{IO}_{2}}$ for oxygen delivery without an endotracheal tube has been demonstrated, placement requires upper-airway topicalization with lidocaine and flexible bronchoscopy, and, sometimes, sedation. ${ }^{5}$ However, there is evidence that $\mathrm{F}_{\mathrm{IO}_{2}}$ measurements at the hypopharynx by using an oral catheter are comparable with measurements at the trachea. ${ }^{4}$ In addition, the oropharyngeal $\mathrm{F}_{\mathrm{IO}_{2}}$ measurement has been obtained via a nasal catheter lubricated with $2 \%$ lidocaine jelly and advanced to the level of the uvula in awake volunteers. ${ }^{3}$

The objective of this study was to determine if $\mathrm{F}_{\mathrm{IO}_{2}}$ at the lips is significantly different than the concentration at the oropharynx when delivered via different oxygen mask designs. These data would provide more accurate information for perioperative clinicians who administer oxygen to subjects who require treatment or prevention of hypoxia and its manifestations, and also would better inform future developers of oxygen delivery systems on the accuracy of methods of measuring $\mathrm{F}_{\mathrm{IO}_{2}}$.

Table 1. Reported $\mathrm{F}_{\mathrm{IO}_{2}}$ Delivery Ranges by Common Oxygen Delivery Devices

\begin{tabular}{lcc}
\hline \multicolumn{1}{c}{ Device } & Flow $(\mathrm{L} / \mathrm{min})$ & $\mathrm{F}_{\mathrm{IO}_{2}}$ \\
\hline Nasal cannula & $1-6$ & $0.24-0.40$ \\
Simple mask & $5-10$ & $0.35-0.50$ \\
Non-rebreather mask & $10-15$ & $0.60-0.80$ \\
OxyMask & $1.5-15$ & $0.25-0.80$
\end{tabular}

$\overline{\text { From References } 1 \text { and } 2 .}$

\section{QUICK LOOK}

\section{Current knowledge}

Previous studies relied on $\mathrm{F}_{\mathrm{IO}_{2}}$ measured at the lips to determine the amount of $\mathrm{O}_{2}$ available when flow is administered $(15 \mathrm{~L} / \mathrm{min})$ oxygen via a mask. It remains unknown whether the $\mathrm{F}_{\mathrm{IO}_{2}}$ measured at the lips differs from the $\mathrm{F}_{\mathrm{IO}_{2}}$ measured at the oropharynx and whether different mask systems perform differently with regard to the $\mathrm{F}_{\mathrm{IO}_{2}}$ levels achieved in the airways.

\section{What this paper contributes to our knowledge}

We found that the average $\mathrm{F}_{\mathrm{IO}_{2}}$ measured at the lips was significantly higher than the average $\mathrm{F}_{\mathrm{IO}_{2}}$ measured at the oropharynx for the OxyMask system and the nonrebreather mask; samples from the closed mask and the simple mask were comparable between the oropharynx and the lips; however, the simple mask did not achieve as high $\mathrm{F}_{\mathrm{IO}_{2}}$ concentrations at high oxygen flows. Therefore, measuring $\mathrm{F}_{\mathrm{IO}_{2}}$ at the lips did not necessarily pre$\operatorname{dict} \mathrm{F}_{\mathrm{IO}_{2}}$ at the oropharynx and the discrepancy between these 2 measurements was dependent on the specific design of oxygen delivery system.

\section{Methods}

\section{Subjects}

Healthy adult subjects between 18 and 70 y old were recruited to the study after providing written informed consent by using a protocol approved by the Oregon Health and Science University Institutional Review Board. The study was performed at Oregon Health and Science University, Portland, Oregon. The study planned to enroll 10 subjects. Recruitment was performed with flyers distributed throughout Oregon Health and Science University and e-mails to the staff of the Department of Anesthesiology and Perioperative Medicine, Oregon Health and Science University. The subjects received a $\$ 5$ gift card to the hospital cafeteria. Recruitment and study procedures were performed from August 2015 to February 2016.

At the time of consent, a brief medical history was taken to screen subjects for eligibility. Eligibility requirements for this study were male and female volunteers between the ages of 18 and 70 years with an American Society of Anesthesiologists (ASA) status of I, II, or III. The subjects also needed to be able and willing to provide informed consent in English. Volunteers were excluded from the study if they had one or more of the following: acute cardiopulmonary disease, as defined by a blood pressure $>150 / 90 \mathrm{~mm} \mathrm{Hg}$, heart rate $>120$ beats/min, and room 
air oxygen saturation $<92 \%$; allergy to lidocaine or adhesive tape; history or physical examination finding of nasal polyps; on oral or parenteral anticoagulants (other than aspirin) at the time of the study; history of frequent nosebleeds; symptoms of nasal congestion at the time of the study; physical examination findings of rales or wheezing; or facial hair that prevented forming a seal with an anesthesia mask. Pregnant women and decisionally impaired adults were excluded. The subjects were required to remain nil per os at least $6 \mathrm{~h}$ before the study to reduce the risk of aspiration on triggering a gag reflex by insertion of the nasal catheter into the oropharynx.

\section{Protocol}

The study took place after hours in a fully equipped operating room (eg, evenings and weekends) to avoid conflict with surgical patient care. Gas analyzers were checked and calibrated before starting the study procedures. This study used an unblinded crossover design with 4 mask systems in 5 different conditions; $\mathrm{F}_{\mathrm{IO}_{2}}$ was measured both at the lips and at the oropharynx for each subject. $\mathrm{F}_{\mathrm{IO}_{2}}$ measurements were collected when applying each of the 4 mask systems sequentially. The subjects were first exposed to room air (no mask), followed by (1) the simple mask system (AirLife, Ref 001201, CareFusion Corp, Yorba Linda, California), (2) a non-rebreather mask system (AirLife, Ref 001203), (3) the OxyMask system, and (4) a closed mask system with a Jackson-Rees circuit (Vital Signs flow-inflating tail-end valve transport circuit, CareFusion). The subjects were asked to breathe normally. To reproduce conventional conditions, no instructions were given for nasal or mouth breathing because unmonitored patients in normal hospital settings are not typically given such instructions.

$\mathrm{F}_{\mathrm{IO}_{2}}$ was first measured at the oropharynx on room air to determine if a consistent measurement could be obtained with the positioning of the oropharyngeal catheter for each subject. $\mathrm{F}_{\mathrm{IO}_{2}}$ was not measured at the lips for room air in the absence of a device; this would provide no contributory information other than the known concentration of oxygen in room air at sea level (ie, 21\%). When a mask system was applied to a subject, the oxygen was set at a flow of $15 \mathrm{~L} / \mathrm{min}$ for $2 \mathrm{~min}$ before measurements were collected to allow for equilibration of inspired oxygen levels inside the mask. For each mask system, $\mathrm{F}_{\mathrm{IO}_{2}}$ was measured first at the lips and then at the oropharynx for 15 consecutive breaths. The mean of the 15 breath $\mathrm{F}_{\mathrm{IO}_{2}}$ measurements were collected for each subject. There were 8 treatment combinations for the 2 locations and 4 mask system conditions plus one room air measurement at the oropharynx. This yielded 9 repeated measurements per subject. The averaged $\mathrm{F}_{\mathrm{IO}_{2}}$ measurements were then used to investigate for differences in the experimental condi-

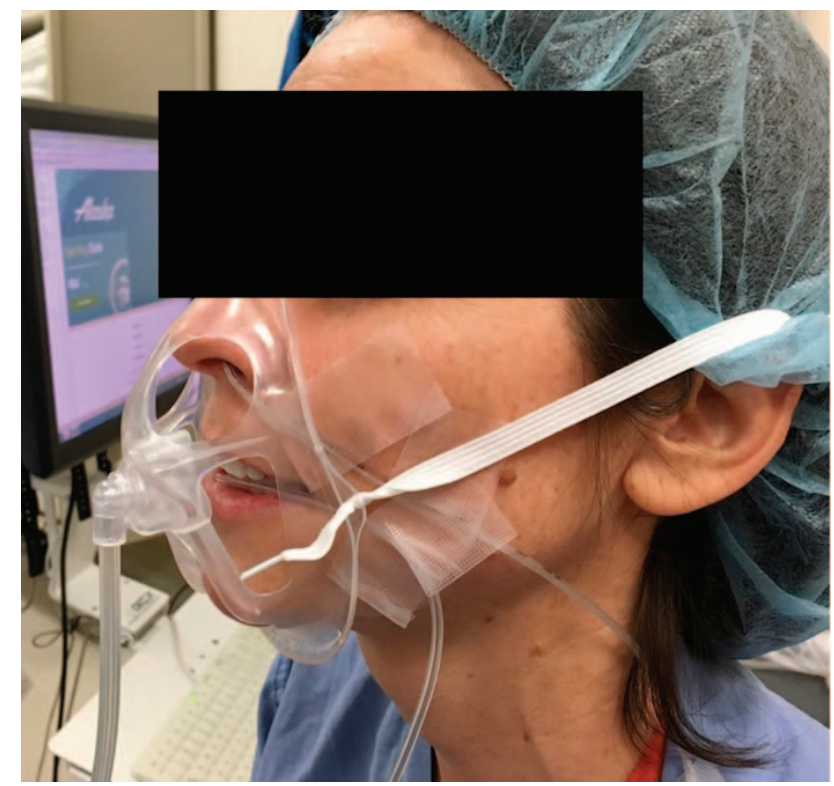

Fig. 1. Experimental setup with the subject wearing the OxyMask. Sampling lines are taped at the lips and oropharynx (through the nostril).

tions. We allowed for a washout period of at least $90 \mathrm{~s}$ between the different treatment combinations in which the subjects breathed room air while the next mask system was being prepared. The study procedures required between 60 and 90 min for each subject to complete the experiment. To minimize extraneous sources of error and variation, the experimental protocol and procedures for data collection were performed by a single, trained research assistant. All procedures were performed with the subject in the seated, upright position.

At the start of the study, 2 sampling lines were connected to a precalibrated gas analyzer (General Electric Datex-Ohmeda, Boston, Massachusetts), with one sampling line taped at the subject's lips and the other attached to a 10 French suction catheter (AirLife Tri-Flo, CareFusion) that was fed through a nostril into the oropharynx (Fig. 1). Protocol placement of the oropharyngeal catheter was similar to that described by Wettstein et al. ${ }^{3}$ Oropharyngeal placement was confirmed by visual inspection of the catheter behind the uvula with the mouth opened and the subject saying "Ah"; if the catheter could not be seen, then placement was confirmed by the gag reflex and a catheter depth of at least $10 \mathrm{~cm}$.

\section{Statistical Analysis}

Statistical analysis was performed to account for the crossover design and repeated measures correlation (within subject) by using a linear mixed effects regression model. We accounted for the repeated measures correlation by 
Table 2. Estimated Mean $\pm \mathrm{SD} \mathrm{F}_{\mathrm{IO}_{2}}$ Levels for the Mask Types and Measurement Location

\begin{tabular}{|c|c|c|c|c|c|}
\hline \multirow{2}{*}{ Device and/or Mask } & \multicolumn{2}{|c|}{$\mathrm{F}_{\mathrm{IO}_{2}}$ by Location } & \multicolumn{3}{|c|}{ Pairwise Differences in $\mathrm{F}_{\mathrm{IO}_{2}}$} \\
\hline & Lips $($ mean $\pm \mathrm{SD})$ & Oropharynx $($ mean $\pm \mathrm{SD})$ & Lips-Oropharynx (mean \pm SE) & $95 \% \mathrm{CI}$ & $P$ \\
\hline Room air & NA & $0.21 \pm 0.004$ & NA & NA & NA \\
\hline Simple mask & $0.67 \pm 0.12$ & $0.62 \pm 0.11$ & $0.04 \pm 0.03$ & -0.01 to 0.10 & .12 \\
\hline Non-rebreather mask & $0.86 \pm 0.09$ & $0.76 \pm 0.10$ & $0.09 \pm 0.03$ & $0.04-0.15$ & .001 \\
\hline OxyMask & $0.81 \pm 0.07$ & $0.51 \pm 0.07$ & $0.30 \pm 0.03$ & $0.25-0.36$ & $<.001$ \\
\hline Closed mask & $0.97 \pm 0.01$ & $0.97 \pm 0.02$ & $0.004 \pm 0.03$ & -0.05 to 0.06 & .89 \\
\hline \multicolumn{6}{|c|}{$\begin{array}{l}\text { Estimated pairwise differences, SEs, CIs, hypothesis tests ( } p \text { values) were derived from fitted regression model } \\
\text { SE }=\text { standard error } \\
\text { NA }=\text { not applicable }\end{array}$} \\
\hline
\end{tabular}

including a single, subject-level, random effect. There were 2 model factors: a 4-level factor for the mask system (ie, 1, simple mask; 2, non-rebreather; 3, OxyMask; 4, closed mask), and a 2-level factor for line location (1, oropharynx; 2, lips). The primary aims of this investigation were to determine whether (1) there were clinically relevant differences in mean $\mathrm{F}_{\mathrm{IO}_{2}}$ levels by line location and (2) by mask system, and (3) to determine if the associations between mean $\mathrm{F}_{\mathrm{IO}_{2}}$ measurements and line location are different across each of the 4 mask systems. We used likelihood ratio tests to evaluate the 3 aims listed above.

Specifically, we computed likelihood ratio statistics by comparing a saturated model with different "reduced models" that omit the factor or factors of interest. For each of the 4 mask systems, we performed pairwise comparisons between the line locations. The hypothesis tests and associated $P$ values did not account for multiple comparisons; one could conservatively adjust for multiple testing by using a Bonferroni adjustment by multiplying the pairwise hypothesis $P$ values by 4 (ie, the number of pairwise comparisons) to provide a bound for an overall significance level. All statistical tests and $P$ values were 2 -sided. Also, the condition room air was not included in the formal analysis of the mask systems because it provided no useful information in comparing the possible associations of mean $\mathrm{F}_{\mathrm{IO}_{2}}$ and line location and mask system. We, however, provided descriptive summaries to show the inherent viability of the method of measurement (Table 2, Fig. 2). Statistical analyses were performed by using Stata version 15.1 (StataCorp, College Station, Texas) and $\mathrm{R}$ version 3.5.1 (R Foundation for Statistical Computing, Vienna, Austria) statistical software packages.

\section{Power and Sample-Size Considerations}

With a significance criterion of 0.05 and within-subject $\mathrm{F}_{\mathrm{IO}_{2}} \mathrm{SD}$ of $3.5 \%, 10$ subjects would provide $>90 \%$ power

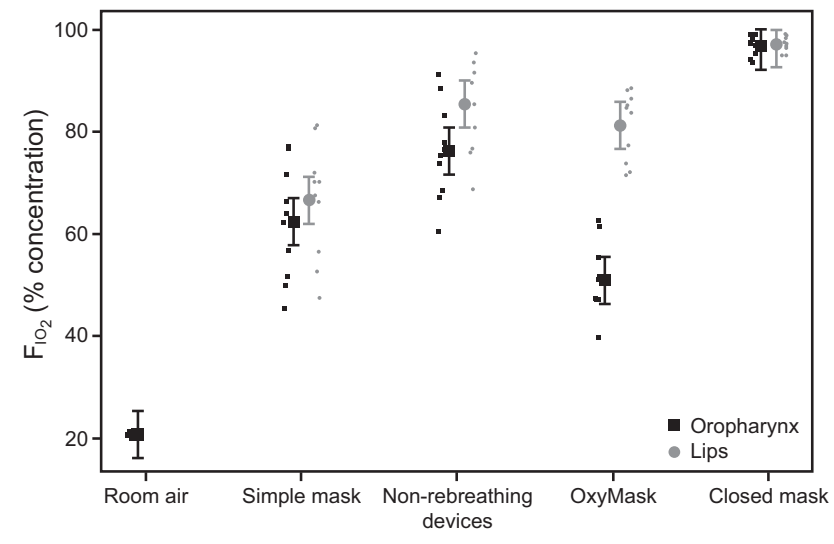

Fig. 2. Mean $\mathrm{F}_{\mathrm{IO}_{2}}$ concentrations by breathing device and sampling location. Error bars show $95 \% \mathrm{Cls}$. Tiny squares and dots correspond to $\mathrm{FiO}_{2}$ measurements for the respective sampling locations.

to detect a mean difference of $10 \% \mathrm{~F}_{\mathrm{IO}_{2}}$ for the crossover design specified. This article adheres to the Transparent Reporting of Evaluations with Non-randomized Designs (TREND) guidelines. ${ }^{8}$

\section{Results}

A total of 14 subjects were invited to participate; 2 subjects were ineligible because of improper nil per os status, and, in 2 additional subjects, the gas analyzer seemed to be poorly calibrated. The improper calibration was not recognized until the measurement with the closed mask did not achieve $\mathrm{F}_{\mathrm{IO}_{2}}$ values $>0.87-0.88$ at the lips and $>0.84$ 0.85 at the oropharynx, which were lower than what would be expected in anesthesia practice in a healthy subject. Therefore, 10 subjects successfully completed the study and were included in the final analysis. None of the $10 \mathrm{sub}-$ jects included in the final analysis experienced adverse events or withdrew from the study, thus the procedures were well tolerated. The oropharyngeal catheter was visualized behind the uvula for 3 of the subjects; placement for 
Table 3. Results of Linear Mixed Effects Regression Model by Examining the Device Effect and the Location Effect, and Their Interaction

\begin{tabular}{lcc}
\hline \hline \multicolumn{1}{c}{ Likelihood Ratio Tests } & Chi-Square Test & $P$ \\
\hline Device/mask effect & 137.9 & $<.001$ \\
Location effect & 74.6 & $<.001$ \\
Location by device interaction & 48.3 & $<.001$
\end{tabular}

Degrees of freedom for device/mask effect (6), location effect (4), device interaction (3)

the other subjects was confirmed by gag reflex and catheter depth.

Mean $\mathrm{F}_{\mathrm{IO}_{2}}$ estimates from our statistical model for the combinations of oxygen mask systems and location of measurement are provided in Figure 2 and Table 2. The mean $\mathrm{F}_{\mathrm{IO}_{2}}$ at $15 \mathrm{~L} / \mathrm{min}$ oxygen at the lips was highest with the closed mask $(0.97,95 \%$ CI $0.92-1.00)$, followed by the non-rebreather mask $(0.86,95 \%$ CI $0.81-0.90)$, OxyMask $(0.81,95 \%$ CI $0.76-0.86)$, then the simple mask $(0.67$, 95\% CI 0.62-0.71) (Table 2). The mean $\mathrm{F}_{\mathrm{IO}_{2}}$ was generally lower at the oropharynx. Mean oropharyngeal $\mathrm{F}_{\mathrm{IO}_{2}}$ was highest for the closed mask (0.97, 95\% CI 0.92-1.00), followed by the non-rebreather mask $(0.76,95 \%$ CI $0.72-$ $0.81)$, the simple mask $(0.62,95 \%$ CI $0.58-0.67)$, and the OxyMask (0.51, 95\% CI 0.46-0.56). Statistical tests and pairwise differences in $\mathrm{F}_{\mathrm{IO}_{2}}$ mean levels between the sampling locations (ie, lips vs oropharynx) for each mask system are provided in Table 2. Omnibus statistical tests for the mask systems, sampling locations, and test of interaction between mask systems and locations are presented in Table 3.

The analysis revealed that $\mathrm{F}_{\mathrm{IO}_{2}}$ measurements differed significantly between the 2 sampling locations and that these differences were dependent on the oxygen mask system used (likelihood ratio test [interaction] $=48.3$, $P<.001$ ) (Table 3). The mean $\mathrm{F}_{\mathrm{IO}_{2}}$ differences for the oxygen mask systems were also highly significant, independent of the location of measurement (likelihood ratio test $[$ mask system] $=137.9, P<.001)$. The mean $\mathrm{F}_{\mathrm{IO}_{2}}$ difference for the line locations were significantly different, independent of the oxygen mask systems (likelihood ratio test $[$ line $]=74.6, P<.001)$. For the pairwise comparisons between the mean $\mathrm{F}_{\mathrm{IO}_{2}}$ levels at the lips compared with the oropharynx for each of the 4 oxygen mask systems, differences were statistically significant for the OxyMask (mean difference 0.30, 95\% CI 0.25- 0.36; $P<.001$ ), and the non-rebreather mask (mean difference 0.09, 95\% CI 0.04-0.15; $P=.001$ ) (Table 2). The mean $\mathrm{F}_{\mathrm{IO}_{2}}$ measured at the oropharynx was not significantly different from the mean $\mathrm{F}_{\mathrm{IO}_{2}}$ measured at the lips for the simple mask system or the closed mask system.

\section{Discussion}

This study demonstrated that $\mathrm{F}_{\mathrm{IO}_{2}}$ levels at the lips were significantly higher than the $\mathrm{F}_{\mathrm{IO}_{2}}$ levels achieved in the oropharynx when delivering high $\mathrm{O}_{2}$ flow $(15 \mathrm{~L} / \mathrm{min})$ via the OxyMask or the non-rebreather mask. This drop in $\mathrm{F}_{\mathrm{IO}_{2}}$ from the lips to the oropharynx most likely represented a dilutional effect contributed by nasal breathing or entrainment of room air around the mouth. Conversely, when high $\mathrm{O}_{2}$ flows were delivered via the closed mask and the simple mask, these devices produced no relevant $\mathrm{F}_{\mathrm{IO}_{2}}$ drops when comparing the lips with the oropharyngeal locations, with the closed mask achieving the highest $\mathrm{F}_{\mathrm{IO}_{2}}$ concentrations at both locations. Analysis of our data indicated that the degree of $\mathrm{F}_{\mathrm{IO}_{2}}$ decrease from the lips to oropharynx depended on the mask system applied.

This difference was much more pronounced for the opendesigned OxyMask (0.30 $\mathrm{F}_{\mathrm{IO}_{2}}$ absolute difference), then the non-rebreather mask ( $0.09 \mathrm{~F}_{\mathrm{IO}_{2}}$ absolute difference), the simple mask (0.04 $\mathrm{F}_{\mathrm{IO}_{2}}$ absolute difference), and the closed mask $\left(0 \mathrm{~F}_{\mathrm{IO}_{2}}\right.$ absolute difference). Other than those used for administration of positive airway pressure, oxygen masks are designed with an imperfect seal on the face. Our findings indicated that the degree of openness of the OxyMask design resulted in an increase in air entrainment passed the measurement point at the lips and a morepronounced drop in $\mathrm{F}_{\mathrm{IO}_{2}}$ from the lips to the oropharynx than with the simple mask and the closed mask. We demonstrated that $\mathrm{F}_{\mathrm{IO}_{2}}$ measurement with the closed mask was not different between the lips and the oropharynx because there was no air entrainment when applying a high degree of seal.

Alternatively, it is conceivable that nasal breathing with the OxyMask may have led to the decrease in oropharyngeal $\mathrm{F}_{\mathrm{IO}_{2}}$ if the oxygen diffuser did not direct oxygen as effectively toward the nares as it did toward the lips. It would be difficult to determine the uniformity of the distribution of oxygen concentration within an oxygen mask without a perfect seal for different subjects and their own particular mask fit. A situation in which face-mask fitting might not influence oxygen concentration in the oropharynx might be the high-flow nasal cannula that represents an alternative oxygen delivery system to standard oxygen therapy. The high-flow nasal cannula compared with the non-rebreather mask has been shown to improve oxygenation in patients who are critically ill $^{9}$ and reduce re-intubation rates or escalation of oxygen therapy. ${ }^{10}$ The $\mathrm{F}_{\mathrm{IO}_{2}}$ measured at the lips with the OxyMask in our study was essentially the same as the 0.80 value reported by Paul et al ${ }^{2}$ (0.81 in this study), which validated our lip sampling line placement and procedural integrity. However, we documented that the oropharyngeal $\mathrm{F}_{\mathrm{IO}_{2}}$ at $15 \mathrm{~L} / \mathrm{min}$ for the OxyMask was only 0.51 . 
In the clinical setting, reasons for the use of oxygen masks include maintenance therapy, treatment of acute hypoxemia, and preoxygenation before intubation. In anesthesia practice, the most common form of preoxygenation is performed with the closed mask system at high oxygen flows. It should be noted that the only mask system in our study that was able to achieve a mean oropharyngeal $\mathrm{F}_{\mathrm{IO}_{2}}$ of $>0.80$ was the closed mask. Based on these findings, we recommend that clinicians in the anesthesia, critical care, and emergency medicine settings preoxygenate with a closed mask whenever possible because the use of any other mask that does not produce a complete seal likely means accepting a $\mathrm{F}_{\mathrm{IO}_{2}}$ of $<0.80$.

The feasibility of gas sampling is important to consider when performing tests on awake spontaneously breathing subjects. We confirmed that oropharyngeal gas sampling was well tolerated and relatively uncomplicated compared with tracheal gas sampling, which requires bronchoscopy and sometimes sedation. It is also feasible to extrapolate $\mathrm{F}_{\mathrm{IO}_{2}}$ from expired oxygen, ${ }^{11,12}$ although this method requires a longer time to allow for equilibration than measurement of inspired oxygen and relies on assumptions of normal respiratory physiology. We propose that an oropharyngeal catheter is a better tolerated yet accurate alternative to the tracheal catheter. Therefore, by being less invasive, oropharyngeal gas sampling may represent a more practical alternative to tracheal sampling in awake subjects because it can be performed with no sedation and does not require bronchoscopy for confirmation of placement.

We used a crossover design to make it possible to obtain measurements on several different interventions with a relatively small number of subjects. Any crossover study has the potential for a carryover effect between treatments, so a washout period and randomization of the order of the treatments are generally used. For this reason, we used a period of $90 \mathrm{~s}$ of room air after each oxygen mask condition and an additional 2 min of breathing oxygen during each mask condition before measuring $\mathrm{F}_{\mathrm{IO}_{2}}$, and we did not randomize the order of the mask conditions. Because the contribution of expired oxygen to $\mathrm{F}_{\mathrm{IO}_{2}}$ inside a mask should be low in the setting of normal resting minute ventilation (5-8 L/min), physiologic dead-space ventilation, entrainment of room air, and high oxygen flows, we believed that any carryover effect during our study procedure would be negligible. This was consistent with the approach used by Paul et al, ${ }^{2}$ which allowed $90 \mathrm{~s}$ between each treatment period during testing of the OxyMask. A possible limitation of our study was that we did not record concentrations in duplicate. However, we allowed sufficient stabilization time before recording the concentration values.

In this study, our goal was to describe the function of each oxygen mask system at a high flow at 2 different measurement locations. We chose to focus on the $\mathrm{F}_{\mathrm{IO}_{2}}$ delivered by oxygen mask systems rather than measuring $\mathrm{P}_{\mathrm{aO}_{2}}$, which is the true end point of oxygen delivery. $\mathrm{F}_{\mathrm{IO}_{2}}$ is independent of a patient's respiratory mechanics and gas-exchange capability, and, therefore, is a more generalizable descriptor for the function of an oxygen mask system than $\mathrm{P}_{\mathrm{aO}}$.

Because patients who are receiving oxygen therapy are generally not instructed whether to breathe through their nose or their mouth for extended periods of time, we did not instruct our subjects which way to breathe for this study. Therefore, it was difficult to determine the contribution of nasal breathing to the difference between lip and oropharyngeal measurements. However, as far as we are aware, no previous studies tested the effects of nasal breathing versus mouth breathing except in the case of nasal cannulas, so this methodology was not diverging from what has been previously reported. It was previously shown that breathing frequency can affect performance of oxygen delivery devices. ${ }^{13,14}$ In this study, we did not record the breathing frequency. However, our subjects were healthy and instructed to breathe normally. Future inquiry would be helpful to determine the relative contribution of nasal breathing and variability in the breathing frequency on pharyngeal oxygen delivery from an open-faced mask such as the OxyMask. Nevertheless, the results of this study should be generalizable to a population of spontaneously breathing patients.

A potential limitation of our experiment was that the oropharyngeal catheter was visualized behind the uvula for only 3 subjects, so it is conceivable that the placement of the catheter only reached the nasopharynx instead of the oropharynx. Nasopharyngeal placement would result in the $\mathrm{F}_{\mathrm{IO}_{2}}$ measurement being weighted toward the nasal concentration of oxygen rather than the oral oxygen concentration. However, because the gag reflex was successfully triggered for every subject in whom the catheter could not be seen behind the uvula, it is unlikely that the catheter was misplaced.

The power and sample-size considerations were performed for pairwise comparison for the location of $\mathrm{F}_{\mathrm{IO}_{2}}$ measurement, an early key consideration in performing this study. We wanted to consider the effect modification of the devices to the association between $\mathrm{F}_{\mathrm{IO}_{2}}$ levels and measurement location (ie, interaction) but did not power specifically for that test. However, we observed a very strong effect modification, due almost exclusively to the large differences for the OxyMask and its corresponding interaction effect estimates. Even though statistical power would not necessarily be high with a modest sample size and tests of interaction (which typically are low-powered tests), we did observe a highly significant effect modification, and, therefore, there was sufficient statistical power to conclude a significant association. 


\section{Conclusions}

It is important that clinicians have accurate $\mathrm{F}_{\mathrm{IO}_{2}}$ data as a basis for determining which oxygen mask system to use for a particular clinical scenario. This study demonstrated that the measurement of $\mathrm{F}_{\mathrm{IO}_{2}}$ at the lips may result in a significant overestimation of $\mathrm{F}_{\mathrm{IO}_{2}}$ in the oropharynx for oxygen mask systems without a perfect seal on the face. Oropharyngeal gas sampling should be considered the preferred method of $\mathrm{F}_{\mathrm{IO}_{2}}$ measurement because it was more accurate than sampling at the lips. This approach was also well tolerated and should be considered for future studies determining the design features of oxygen delivery devices.

\section{ACKNOWLEDGMENTS}

We thank Lisa Voltolina for her work on the institutional review board submission and Kimberly Voelker for her editorial contribution to completion of the manuscript.

\section{REFERENCES}

1. Kallstrom TJ, American Association for Respiratory Care (AARC). AARC Clinical Practice Guideline: oxygen therapy in adults in the acute care facility - 2002 revision and update. Respir Care 2002; 47(6):717-720.

2. Paul JE, Hangan H, Hajgato J. The OxyMask $\left({ }^{\mathrm{TM}}\right)$ development and performance in healthy volunteers. Medical Devices (Auckl) 2009; 2:9-17.

3. Wettstein RB, Shelledy DC, Peters JI. Delivered oxygen concentrations using low-flow and high-flow nasal cannulas. Respir Care 2005; 50(5):604-609.
4. Schacter EN, Littner MR, Luddy P, Beck GJ. Monitoring of oxygen delivery systems in clinical practice. Crit Care Med 1980;8(7):405409.

5. O'Reilly Nugent A, Kelly PT, Stanton J, Swanney MP, Graham B, Beckert L. Measurement of oxygen concentration delivered via nasal cannula by tracheal sampling. Respirology 2014;19(4):538-543.

6. Kory RC, Bergmann JC, Sweet RD, Smith JR. Comparative evaluation of oxygen therapy techniques. JAMA 1962;179:767-772.

7. Beecroft JM, Hanly PJ. Comparison of the OxyMask and Venturi mask in the delivery of supplemental oxygen: pilot study in oxygendependent patients. Can Respir J 2006;13(5):247-252.

8. Des Jarlais DC, Lyles C, Crepaz N; TREND Group. Improving the reporting quality of nonrandomized evaluations of behavioral and public health interventions: the TREND statement. Am J Public Health 2004;94(3):361-366.

9. Brotfain E, Zlotnik A, Schwartz A, Frenkel A, Koyfman L, Gruenbaum SE, Klein M. Comparison of the effectiveness of high flow nasal oxygen cannula vs. standard non-rebreather oxygen face mask in post-extubation intensive care unit patients. Isr Med Assoc J 2014; 16(11):718-722.

10. Rochwerg B, Granton D, Wang DX, Helviz Y, Einav S, Frat JP, et al. High flow nasal cannula compared with conventional oxygen therapy for acute hypoxemic respiratory failure: a systematic review and meta-analysis. Intensive Care Med 2019;45(5):563-572.

11. Markovitz GH, Colthurst J, Storer TW, Cooper CB. Effective inspired oxygen concentration measured via transtracheal and oral gas analysis. Respir Care 2010;55(4):453-459.

12. Bazuaye EA, Stone TN, Corris PA, Gibson GJ. Variability of inspired oxygen concentration with nasal cannulas. Thorax 1992;47(8): 609-611.

13. Lamb K, Piper D. Southmedic OxyMask(TM) compared with the Hudson RCI(®) Non-Rebreather Mask(TM): safety and performance comparison. Can J Respir Ther 2016;52(1):13-15.

14. Wagstaff TA, Soni N. Performance of six types of oxygen delivery devices at varying respiratory rates. Anaesthesia 2007;62(5):492-503.

This article is approved for Continuing Respiratory Care Education credit. For information and to obtain your CRCE

(free to AARC members) visit www.rcjournal.com

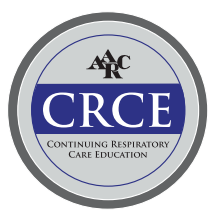

